\title{
Application of GPON in Campus Network
}

\author{
Xiaohui Yang \\ Stute-owned Assets Administration \\ Chifeng University \\ Chifeng City, Inner Mongolia, P.R.China \\ 550623564@qq.com
}

\begin{abstract}
Keywords: GPON(Gigabit Passive Optical Network); Campus Network ; Application
\end{abstract}
\begin{abstract}
The past few years have seen the introduction and wide-spread implementation of passive optical network, first in big cities, followed by use in countryside. With the rapid development increased demand for lower operating costs, making the XPON optical access technology as represented in the international and domestic mainstream operators are widely used. This paper introduces the application of GPON (gigabit passive optical network) in campus network, and the differences with traditional campus network. At the same time, according to practical construction situation of campus network in Chifeng University, analyzes the advantage and disadvantage of GPON in campus network solution, in order to provide some useful new ideas for digital campus construction.
\end{abstract}

\section{Introduction}

Passive Optical Network (PON) is a new technology emerged in the recent years, and it's indisputable that the application of passive optical network is used more and more widely. Passive optical network is an optical point-to-multipoint access network. There are no optical repeaters or other active devices in a PON, hence the name "passive." [1] PONs are designed for local loop transmission rather than long distance and serve to bring fiber closer to the customer in order to obtain higher speed. The development and application of passive optical network experienced different periods such as APON, BPON, EPON and GPON. APON (ATM PON) was the first passive optical network and uses ATM (Asynchronous Transfer Mode) for transport. BPON (Broadband PON) includes APON, Ethernet and video transports. GPON (Gigabit PON), which uses the SONET (Synchronous Optical Networking) frame, is designed to be efficient for packets as well as TDM. BPON and GPON are the ITU-T G.983 and G.984 standards respectively. EPON is the IEEE Ethernet standard for PONs [2].

At present, most campus network structures of colleges and universites in mainland China is consisted of the three layer switching structures, which based-on traditional ethernet model. With the continuous development of information technology, users' requirements on the network bandwidth and stability continue to increase, the traditional ADSL or LAN access has been unable to meet the demand for new business, thereby a number of operators have falled into intense conflict in the "last one mile" of Internet access. Among them, with its large capacity, low attenuation, high bandwidth, anti interference performance, capable of bearing high quality video, features such as green, FTTH (Fiber to the Home) optical access network becomes the new focus. It is in this context, Chifeng University cooperates with Inner Mongolia Chifeng Branch of China Unicom to build digital campus network based-on GPON technology.

\section{GPON technology}

GPON or Gigabit Passive Optical Network is an optical technology based on the industry standard ITU-TG.984x which was ratified in 2003[3]. This technology was originally developed to provide high speed Ethernet services for residential and small business customers. Over the last several years, significant development of new enterprise GPON technology has taken place and now large business 
or government agencies can deploy this high bandwidth, power-saving, green technology in their data networks today.

GPON's passive optical network architecture allows for a very efficient, distributed deployment of Gigabit Ethernet service to the end-user. For most customers, this translates into a high bandwidth desktop availability allowing researchers, scientists, and businesses to quickly perform large data file transfers or run high bandwidth cloud computing data applications.

For network administrators, GPON also allows for a phased in migration approach of voice, data, and video services; including the ability to simultaneously support VoIP(Voice over Internet Protocol ) and POT(Plain old telephone) services over the same fiber. Powerful security measures at the physical layer, data layer, and the end user port greatly reduce or eliminate the potential for Denial of Service (DoS), redirects, or other malicious attacks. Enterprise GPON is also a carrier class technology that provides a high level of Quality of Service (QOS) 99.999\% for those customers with mission-critical requirements.

Today, the acronym is known widely as FTTx (Fiber-to-the-x), where $\mathrm{x}$ can denote different destinations. The most important are: FTTH (home), FTTB (building) and FTTN (node). In FTTH fiber to the home or fiber reaches the subscriber's home or office. In contrast, the fiber ends before FTTB typically in or near the building of subscribers. FTTN at the far ends of fiber subscribers in FTTH and FTTB, typically in the immediate neighborhood. The choice of this or that architecture mainly depends on the unit cost per end user and the type of services it offers the operator.

In a FTTB and FTTN architecture, which is the model that will have more success in Spain in the short term, the fiber optic link is established between a central office and a distribution point in between. From this point of intermediate distribution, access to subscribers of the home or building through technology VDSL2(Very high bit-rate Digital Subscriber Line 2) on the copper pair or WiMax (Worldwide Interoperability for Microwave Access) wireless way. Thus, the laying of fiber can be achieved gradually, in less time and with less cost by reusing the infrastructure of the subscriber.

GPON has long been a favorite approach for the more and more clients, thanks to properties of fiber optic physical security plus high bandwidth. GPON's bandwidth advantages have been eliminated as Ethernet switching has progressed from 1 Gigabit per second (Gbps) to 10 Gbps to now 40 and 100 Gbps. In addition, GPON's physical security afforded by difficulty of tapping fiber optic cabling without detection is not an exclusive GPON attribute, but any and all networks that utilize fiber optic transport, including Ethernet switching. But most importantly, over the years, fiber optic taps have become available with insertion losses as low as $0.5 \mathrm{~dB}$ and lower, making fiber optic cable physical security no more secure than copper [4] .

On the other hand, lower cost cabling, lower power consumption and fewer network devices required all are the GPON's virtue. The use case of GPON is commonly defined as those that require 1 Gbps at the desktop. A case of GPON campus network architecture is presented before we review these claims.

\section{Campus network based-on GPON: taking Chifeng University as an example}

Today, Educational reform and development in China is confronted with hitherto unknown challenge and opportunity. Promoting the modernization of education with the education information and breaking restriction in education development and enhancing educational innovation and change are major strategy choice to accelerate China becoming a powerful nation from a big country of education. China Ministry of Education has given the development target of Education Informatization ten-year development plan in March, 2012. That is, by 2020, basically everyone can enjoy the high quality education resources and information learning environment. Informatization supporting service system of a learning society can be formated and broadband network coverages all levels of all regions and all types of schools, and the levels of education management rise significantly, information technology and education development level significantly improve. 


\subsection{About Chifeng Universities and its Campus Network}

The Chifeng University is approved by the State Ministry of Education in 2003, has more than 40 years of school history with a good reputation for a multidisciplinary undergraduate colleges and universities. It is one of 844 Common Universities published by Ministry of Education of the People's Republic of China in 2012[5]. Chifeng University is located in the famous "Hongshan Culture" birthplace - Chifeng City, Inner Mongolia ,which has a long history culture and the rich local resources.

The University great efforts to strengthen international exchanges and cooperation, to establish good relations of cooperation with the relevant institutions of the United States, the United Kingdom, France, Canada, South Korea, Mongolia and other countries. At present, there are about 200 foreign students from Mongolia, South Korea, Ghana and other countries in Chifeng University.

The running campus network currently was officially opened on October 15, 2002. With the development of Internet an overall transformation had been conducted in 2007. The campus network puts to use the Internet/Intranet architecture and TCP/IP communication protocol. With Gigabit Ethernet technology and three-tier design patterns, such as core layer, distribution layer and access layer. The campus network Drew into Vlan technology and Pvlan technology in order to ensure information security in various departments. The Lenovo networking products, it is advanced at that time, was chosen as the main network equipment. The backbone medium to connect various buildings is fiber optic cable, which starts from core switching machine. The connected medium in one building is unshielded twisted pair (UTP). The campus network accesses Internet via the China Tietong and the outlet bandwidth is $100 \mathrm{M}$.

\subsection{New campus network based-on GPON to be put into use}

Because the existing network resources in Chifeng College has far can't meet the needs of teachers and students. And that, a number of constructions in the campus are being built or planning to build. It is necessary to upgrade the running campus network.

Chifeng University, under the guidance of the concept "Market for Resources", cooperated with China Unicom Chifeng Branch to build "Digital Campus" of Chifeng University in 2011. They would have an all-round cooperation in integrated communication services business, and carry out mutual benefit and win-win, innovation and development.

Campus network system is the operation platform of digital campus, and it is the necessary environment for teaching, science research, management systems, multimedia and other applications. According to different users and their geographical distribution, the design of new campus network is based on GPON technology and combines with the layout of the original network line. The design uses FTTH network structure, and carries out fiber to the home, so as to solve the demand of the users for the network bandwidth. The specific configuration is as follows: the core router of data-control rooms adopts Huawei NE40E, which is the high-end network products launched by Huawei, has a powerful routing capabilities, and support for large routing tables in order to ensure security and stability in the complex routing environment. Switching equipment is Huawei S7706. The OLT (optical line terminal) device provided by Huawei is set in the center computer room, which is a blade access product with high-capacity and all-optical interface. Considering the flexibility in the process of construction, line attenuation and the cost of the project, the design uses a spectral model with the splitters that is 1:32 and connects with the end-user through the ONTs (Optical Network Termination). The specific configuration is shown in Figure 1.

\subsection{Analysis of campus network based-on GPON}

Obviously, the campus network based-on GPON has some advantages compared with the traditional three-tier Ethernet campus network. GPON is positioned to reduce the number of active switching devices in the design of a computer network. The best practice of campus computer network architecture used to be access, distribution and core. Note that new price points and inter-Ethernet switching protocols that eliminate spanning tree protocol (STP)are allowing access and distribution layers to be collapsed into one, offering choice of a two- or three-tier campus network design. 


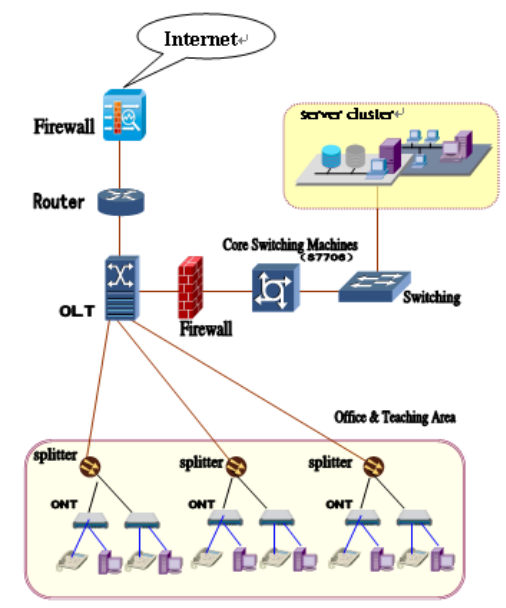

Fig. 1. Campus network based-on GPON

In three-tier network architecture, at each tier, active Ethernet switches forward packets to their destination, affording a mesh of traffic flows to traverse the network. Endpoints or desktops connected on a common access switch may communicate directly with each other without the need for packets to travel to distribution and/or core switches, reducing latency via efficient forwarding. This is especially important for real-time communications, such as Voice over IP and video conferencing, which are increasingly peer-to-peer flows.

In addition, Ethernet vendors have been investing in network services such as security, quality of service, Power over Ethernet, application monitoring, power consumption monitoring, video enablement, etc. Many of these network services start at the access tier, thanks to software features embedded within Ethernet access switches and traverse the entire campus network.

GPON in the campus replaces this three-tier architecture with a two-tier fiber optic network by eliminating active access and distribution Ethernet switches with passive optical devices, which consist of the following things.

-OLT or Optical Line Terminal: The OLT aggregates all optical signals from vendor dependent ONTs into a single multiplexed beam of light which is then converted to an electrical signal, formatted to Ethernet packet type standards and presented to a core Ethernet switch for layer 2 or 3 forwarding. A typical OLT supports some $36 / 72$ ports, each port being a fiber optic cable that has been multiplexed via a splitter in the upstream direction. A typical OLT port supports 32 ONTs, however technical literature states 128 ONTs per OLT port. All traffic from endpoints is multiplexed up to a core switch for layer 2 or 3 forwarding. While some OLT vendors are starting to offer VLAN-aware products, this does not avoid the hairpinning of traffic where the core switch provides forwarding. OLT possesses approximately 200 Gbps of switching capacity.

-Splitters: Optical splitters are used to fan in or multiplex, usually 1:32, fiber optic signals to a single upstream fiber optical cable. Careful consideration of power budget analysis needs to be performed to assure adequate optical signal strength.

-ONT or Optical Network Termination: The ONT connects desktops into the GPON network and primarily provides the optical to electrical signal conversion. ONTs also provide AES (American Electrochemical Society) encryption via ONT key.

The GPON campus network has the following attributes:

-The Physical GPON network is a hub and spoke architecture that multiplexed upstream and broadcast downstream traffic flows.

-The logical GPON network is a single layer 2 broadcast domains as layer 3 services are provided in the core Ethernet switch.

-Another attribute of GPON is its alleged power consumption benefit being some $80 \%$ less than a three-tier network. But its reliability still needs to be confirmed. 


\section{Some questions need further discussion}

Although the campus network based-on GPON has some distinct advantage compared with the traditional Ethernet campus network, it is still a contentious issue that GPON technology is applied in campus network. In the end it is good or bad? Various scholars have given different answers for this question. Steven Pollock, a Sr. Systems Engineering Manager and a 15-year IT Veteran, is one of them. In the end of his Blog article GPON in the Campus Network - A Misuse of Perfectly Good Technology? Steven said, all this adds up to GPON being a poor choice in the Campus when you look at the Total Cost of Ownership (TCO) even though the initial acquisition costs might be lower for the hardware itself. When you look under the covers, the real price is quite high for GPON in terms of a "lack of flexibility, greater power consumption (certainly not green), limited network capacity, upgrades are system-wide events, troubleshooting tools and skilled technicians are limited and lacking, and multiple single points of failure exist." He went on to say, with the Ethernet market being tens of billions of dollars, research and development is assured while competition provides the motivation for innovation and feature enhancement. Maybe, An Ethernet campus network is a safe investment.

The author did not carry out detailed calculations for the power consumption of GPON network vs. Ethernet in Chifeng University. But campus network based-on GPON has shown some problems, of course, of which there are some human factors, in the process of the construction of campus network. Maybe, the effect of GPON applied to the campus network is not as good as people's imagination. Which is better, GPON or Gigabit Ethernet? Some relevant questions need further research in practice.

\section{Summary}

Campus network has been widely used in the field of education, and it plays an active role in education more and more important. GPON campus network is becoming one of the prevailing technologies of digital campus with the popularization of FTTx. In recent years, with the protocol standards further perfecting of network and the cost performance ratio of products improving, GPON network has been applied in the campus gradually, and plays a more important role in the campus, the emergence of network teaching mode will make the GPON network more widely applied in education.

Just like any other new thing, the pros and cons of GPON campus network also need to be demonstrated in practice. A better campus network building programs will be put forward in the practical application. We are hopeful.

\section{References}

[1] JIANG Yong-zhong, ZHANG Ming-lun, ZHANG Qiu-sheng, "GPON: Technology of the Next Access Network", Optical Communication Technology, 2005(1).

[2] ITU-T. Recommendation G.984.2. Gigabit-capable Passive Optical Networks (GPON): Transmission convergence layer Specification. 2004.

[3] ITU-T. Recommendation G.984.1. Gigabit-capable Passive Optical Networks(GPON): General characteristics. 2003.

[4] Steven Scott Gorshe, Overview and Comparison of the IEEE EPON and ITU-T GPON Protocols. China Communications. 2007, 4(2).

[5] Ministry of Education of the People's Republic of China, http://www.moe.edu.cn/publicfiles/business/htmlfiles/moe/moe_122/201206/137993.html, retrieved on August 24, 2012. 